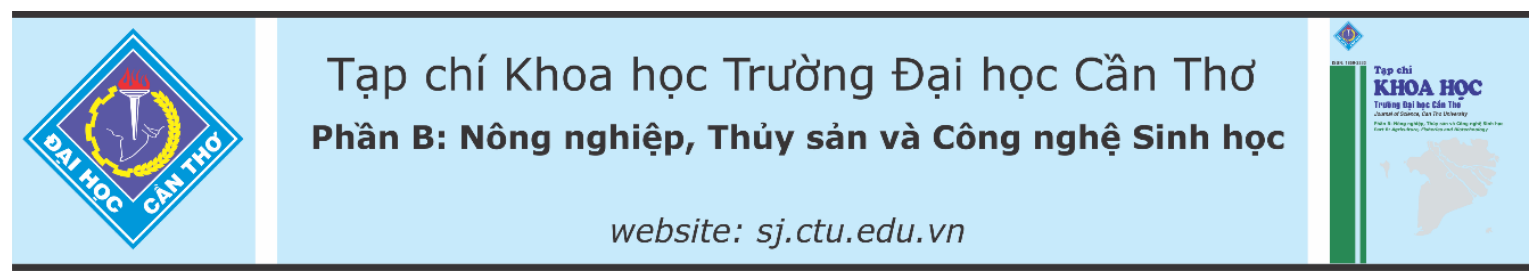

DOI:10.22144/ctu.jvn.2021.015

\title{
ẢNH HƯỞNG CỦA NHIẸTT ĐỘ ĐẾN ĐộNG HỌC QUÁ TRİNH SÂYY CÀ CHUA BI ĐEN (Solanum lycopersicum cv. OG) TRONG ĐIỀU KIỆN CHÂN KHÔNG
}

\author{
Hồ Thị Ngân Hà ${ }^{1,2^{*}}$ và Nguyễn Minh Thủy ${ }^{2}$ \\ ${ }^{1}$ Khoa Nông nghiệp và Tài nguyên thiên nhiên, Truờng Đại học An Giang, Đại học Quốc gia Thành phố Hồ \\ Chi Minh \\ ${ }^{2}$ Bộ môn Công nghệ thực phẩm, Khoa Nông nghiẹp, Truoòng Đại học Cần Tho' \\ "Nguoòi chịu trách nhiệm về bài viết: Hồ Thị Ngân Hà (email: htnha@agu.edu.vn)
}

\section{Thông tin chung:}

Ngày nhận bài: 30/09/2020

Ngày nhận bài sủa: 05/12/2020

Ngày duyệt đăng: 27/02/2021

Title:

Influence of air temperature on the vacuum drying kinetics of black cherry tomatoes (Solanum lycopersicum cv. $O G)$

\section{Tù khóa:}

Cà chua bi đen, độ khuếch tán ẩm, mô hình toán hoc, năng lương hoạt hóa, sấy chân không

\section{Keywords:}

Activation energy, black cherry tomato, mathematical model, moisture diffusivity, vacuum drying

\section{ABSTRACT}

Vacuum drying is an advanced method widely used in the processing of dried fruit and vegetable products. In this study, the effect of four temperatures $\left(40^{\circ} \mathrm{C}\right.$, $50^{\circ} \mathrm{C}, 60^{\circ} \mathrm{C}$, and $70^{\circ} \mathrm{C}$ ) at a constant vacuum level of $-700 \mathrm{mmHg}$ (corresponding to an absolute pressure of $60 \mathrm{mmHg}$ ) on the kinetics of moisture ratio change of black cherry tomatoes (Solanum lycopersicum cv. OG) were investigated. The suitable model for describing the vacuum drying process was chosen by fitting eight commonly used drying models (Lewis, Page, modified Page, Henderson and Pabis, logarithmic, two-term, two-term exponential, modified Henderson and Pabis). The effective moisture diffusivity and activation energy were calculated using the Fick's diffusion equation. The results showed that increasing drying temperature accelerated the vacuum drying process and the goodness of fit tests indicated that the suggested logarithmic model gave the best fit to experimental data among the eight tested drying models. The everage effective moisture diffusivity values varied from $3,9028.10^{-10}$ to $1,7580.10^{-9} \mathrm{~m}^{2} / \mathrm{s}$ over the temperature range. The temperature dependence of the effective moisture diffusivity for the vacuum drying of the black cherry tomato samples was satisfactorily described by an Arrhenius-type relationship with activation energy value of $38.69 \mathrm{~kJ} / \mathrm{mol}$ within 40 to $70^{\circ} \mathrm{C}$.

\section{TÓM TẮT}

Sấy chân không là một phuoong pháp tiên tiến được sủ dụng rộng rãi trong chế biến các sản phẩm rau quả. Trong nghiên cứu này, ảnh hưởng của bốn nhiệt độ sấy khác nhau $\left(40^{\circ} \mathrm{C}, 50^{\circ} \mathrm{C}, 60^{\circ} \mathrm{C}\right.$ và $\left.70^{\circ} \mathrm{C}\right)$ ở mức chân không cố định là -700 mmHg (tuoong úng với áp suất tuyệt đối $60 \mathrm{mmHg}$ ) đến động học biến đổi tỷ lệ ẩm của trái cà chua bi đen (Solanum lycopersicum cv. OG) đã được khảo sát. Tám mô hình sấy thông dụng (Lewis, Page, Page điều chỉnh, Henderson và Pabis, logarit, hai tham số, hàm mũ hai tham số, Henderson và Pabis điều chỉnh) được kiềm tra để chọn ra mô hình phù hợp. Độ khuếch tán ẩm hiệu quả và năng luợng hoạt hóa được tính toán bằng phuoong trình khuếch tán Fick. Kết quả cho thấy nhiệt độ sấy tăng đã thúc đẩy quá trình sấy chân không diễn ra nhanh hơn và mô hình logarit được chưng minh là phù hợp nhất với các dũ liệu thực nghiệm trong số tám mô hình thư nghiệm. Giá trị độ khuếch tán ẩm hiệu quả dao động tù 3,9028.10 $10^{-10}$ đến $1,7580.10^{-9} \mathrm{~m}^{2} / \mathrm{s}$ trong phạm vi nhiệt độ khảo sát. Sư phụ thuộc nhiệt độ của độ khuếch tán ẩm hiệu quả tuân theo phuoong trình Arrhenius với giá trị năng luợng hoạt hóa là $38,69 \mathrm{~kJ} / \mathrm{mol}$ trong khoảng nhiệt độ $40-70^{\circ} \mathrm{C}$. 


\section{1. ĐẶT VẤN ĐỀ}

Cà chua bi đen là loại cà chua mới xuất hiện ở Việt Nam trong những năm gần đây và đang được người tiêu dùng quan tâm. Lycopene là loại carotenoid dồi dào nhất (chiếm khoảng $80-90 \%$ tổng lượng carotenoid) và có khả năng chống oxy hóa cao nhất trong cà chua (Alda et al., 2009). Các nghiên cứu dịch tễ học đã chứng minh được sắc tố đỏ này có khả năng làm giảm nguy cơ mắc các bệnh mãn tính, đặc biệt là ung thư tuyến tiền liệt (Ford and Erdman, 2012), đóng vai trò quan trọng trong việc ngăn ngừa bệnh tim mạch (Mordente et al., 2011), giúp giảm nguy cơ tử vong do bệnh Alzheimer ở người trưởng thành (Min \& Min, 2014), có lợi trong việc điều trị bệnh Parkinson và các bất thường thần kinh khác bằng cách bảo vệ chống lại stress oxy hóa (Kaur et al., 2011). Ngoài carotenoid, cà chua còn chứa các thành phần chống oxy hóa khác như vitamin $C$ và các hợp chất phenolic có khả năng ức chế các loại oxy hoạt động gây ra nhiều bệnh nguy hiểm (Ilahy et al., 2009). Đáng chú ý là hàm lượng các hợp chất phenolic và sắc tố carotenoid, đặc biệt là lycopene trong cà chua bi đen được tìm thấy còn cao hơn so với các giống cà chua đỏ (Zhang et al., 2018). Đặc biệt, cà chua bi đen còn sản sinh ra một thành phần độc đáo là anthocyanin tập trung chủ yếu ở phần vỏ bên ngoài mà các loại cà chua đỏ không có (Li et al., 2011). Anthocyanin đã được chứng minh là có liên quan đến nhiều lợi ích sức khỏe, làm giảm sự tăng sinh tế bào ung thư, bảo vệ chống lại bệnh tim mạch cũng như ngăn ngừa béo phì và tiểu đường (Lila, 2004). Do những lợi ích về sức khỏe nên trái cà chua có thể được sử dụng như rau tươi hoặc chế biến thành nhiều sản phẩm phổ biến (Zhang et al., 2018) như sốt cà chua, nước ép cà chua, bột cà chua, cà chua sấy dẻo, cà chua ngâm giấm.

Sấy khô là một trong những phương pháp bảo quản thực phẩm quan trọng và lâu đời nhất (Michailidis and Krokida, 2014). Việc loại bớt ẩm từ nguyên liệu giúp ngăn cản sự phát triển và sinh sản của các vi sinh vật gây hư hỏng, làm chậm hoạt động của enzyme và giảm thiểu nhiều phản ứng xấu liên quan đến ẩm (Wu et al., 2007). Mặc dù sấy có hiệu quả kéo dài thời gian bảo quản nông sản nhưng sự giảm chất lượng về cảm quan và dinh dưỡng là không thể tránh khỏi trong quá trình sấy khô truyền thống do những biến đổi không mong muốn về cấu trúc và đặc điểm sinh hóa (Wu et al., 2007).

So với sấy trong điều kiện khí quyển thông thường, sấy chân không có một số đặc điểm nổi trội như tốc độ sấy cao hơn, nhiệt độ sấy thấp hơn và môi trường ít oxy hơn dẫn đến chất lượng và giá trị dinh dưỡng của sản phẩm sấy giữ được tốt hơn (Thorat et al., 2012). Sấy chân không hoạt động ở áp suất thấp hơn, điểm sôi của nước giảm nên sự bay hơi ẩm diễn ra ở nhiệt độ thấp hơn, điều này giúp bảo vệ các thành phần nhạy cảm với nhiệt có trong thực phẩm (Thorat et al., 2012). Ngoài ra, môi trường ít oxy trong quá trình sấy chân không sẽ ngăn cản được các phản ứng oxy hóa (Thorat et al., 2012). Sấy chân không đã được áp dụng để sấy các loại trái cây và rau quả khác nhau như xoài (Jaya and Das, 2003), cà rốt và bí đỏ (Arevalo-Pinedo and Murr, 2007), cà tím (Wu et al., 2007), gừng (Thorat et al., 2012) với chất lượng sản phẩm vượt trội.

Nghiên cứu động học thường được sử dụng để mô tả cơ chế của quá trình truyền khối trong khi sấy và chịu ảnh hưởng đáng kể bởi điều kiện sấy (Giri and Prasad, 2007). Mô hình động học sấy là rất cần thiết cho việc thiết kế thiết bị, tối ưu hóa quá trình và cải thiện chất lượng sản phẩm (Giri and Prasad, 2007). Do đó, mục tiêu của nghiên cứu là hiểu biết cơ bản về động học thay đổi tỷ lệ ẩm của cà chua bi đen trong quá trình sấy chân không ở các nhiệt độ khác nhau và chọn mô hình sấy phù hợp trong số các mô hình được thực hiện.

\section{PHƯƠNG PHÁP NGHIÊN CÚU}

\subsection{Chuẩn bị mẫu cà chua}

Hạt giống cà chua bi đen (Solanum lycopersicum cv. OG) được cung cấp từ cửa hàng hạt giống F1508 (Thành phố Hồ Chí Minh) và trồng tại nhà lưới của cơ sở sản xuất và kinh doanh Nam Long (Vĩnh Long). Trái cà chua được thu hoạch ở độ chín hoàn toàn (32 ngày sau khi đậu trái) khi đường kính trái đạt $25,11 \pm 1,83 \mathrm{~mm}$, khối lượng trái $22,25 \pm 1,28 \mathrm{~g}$; hàm lượng chất khô hòa tan tổng và giá trị $\mathrm{pH}$ tương ứng là $6,17 \pm 0,12^{\circ}$ Brix và $4,43 \pm 0,06$; độ cứng trái $899 \mathrm{~g}$ (trái được đo độ cứng bằng thiết bị Rheo Tex (SD 700, Sun Science, Nhật), sử dụng đầu đo hình trụ đầu phẳng có đường kính $1 \mathrm{~cm}$ đo lực cần thiết nén thẳng vào giữa quả với quãng đường di chuyển $4 \mathrm{~mm}$ ). Loại bỏ những trái bị hư hỏng hoặc khuyết tật. Cà chua sau khi thu hoạch được cho vào hộp nhựa $\mathrm{PVC}$ (polyvinyl clorua) và sau đó cho vào thùng carton để tránh làm tổn thương quả, cả hộp nhựa $\mathrm{PVC}$ và thùng carton đều có đục lỗ để tạo sự thông thoáng, vận chuyển về phòng thí nghiệm của Bộ môn Công nghệ Thực phẩm, Trường Đại học Cần Thơ trong vòng 1 giờ. Cà chua được rửa sạch và ngâm trong nước có sục khí ozone trong 15 phút bằng thiết bị tạo ozone 2 vòi (Z755, Việt Nam, khả năng tạo ozone $80,4 \mathrm{mg} / \mathrm{h}$ ) để tiêu diệt các vi sinh vật trên bề mặt, khối lượng mẫu $1,5 \mathrm{~kg}$, tỷ lệ giữa 
nguyên liệu và nước là 1:2 (Ha and Thuy, 2020a). Quá trình xử lý chân không sau đó được thực hiện trong hệ thống kín được kết nối với bơm chân không (Rocker 400, Laftech, Úc) để thay thế các chất khí bên trong trái bằng chất lỏng nhằm tăng độ dẫn nhiệt cho quá trình chần tiếp theo. Độ chân không và thời gian xử lý được chọn lần lượt là $620 \mathrm{mmHg}$ và 22 phút với tỷ lệ nguyên liệu và nước là $1: 1$, sau đó, chân không được phá bỏ, mẫu được lấy ra khỏi thiết bị và giữ ở điều kiện khí quyển trong 15 phút $\quad$ Ha and Thuy, 2020b). Trái sau khi xử lý chân không được ngâm trong dung dịch $\mathrm{CaCl}_{2} 2,08 \%$ ở nhiệt độ $62^{\circ} \mathrm{C}$ trong 23 phút trước khi thực hiện quá trình chần trong nước ở nhiệt độ $90^{\circ} \mathrm{C}$ trong 1 phút nhằm cải thiện độ cứng của trái. Quá trình xử lý $\mathrm{CaCl}_{2}$ và chần đều được thực hiện bằng cách cho $1 \mathrm{~kg}$ mẫu vào trong rổ lưới bằng inox hình chữ nhật (dài 25,5 $\mathrm{cm}$; rộng $10 \mathrm{~cm}$; cao $6,5 \mathrm{~cm}$ với các lỗ vuông kích thước $0,5 \mathrm{~cm}$ ) và ngâm vào bể điều nhiệt (Rex C-90, Memmert, Đức), trong đó tỷ lệ nguyên liệu và dung dịch là 1:2. Sau đó, trái được làm lạnh nhanh bằng nước lạnh $\left(10^{\circ} \mathrm{C}\right)$ trong 1 phút để ngăn chặn sự phá hủy bởi nhiệt, làm ráo và dùng kim xâm lỗ nhỏ trên bề mặt trái (đường kính lỗ $1 \mathrm{~mm}$, mật độ 20 lỗ/ $\mathrm{cm}^{2}$ ) do cà chua có lớp vỏ sáp gây khó khăn cho quá trình truyền khối giữa quả và dung dịch ưu trương. Quá trình thẩm thấu có ứng dụng xử lý chân không được thực hiện bằng cách ngâm trong dung dịch đường saccharose ở nồng độ $59^{\circ}$ Brix, độ chân không 625 mmHg trong 11 phút, sau đó, chân không được phá bỏ, mẫu được lấy ra khỏi thiết bị và trái cà chua vẫn tiếp tục được ngâm trong dung dịch đường saccharose này ở áp suất khí quyển để thực hiện quá trình tách nước thẩm thấu trong 4 giờ. Các thông số trên đây đều là kết quả thu được từ các nghiên cứu đã được thực hiện bởi nhóm tác giả.

\subsection{Bố trí thí nghiệm}

Cà chua bi đen sau khi thẩm thấu trong dung dịch đường được sấy bằng tủ sấy chân không (WiseVen VO-30, Scilab, Hàn Quốc) có kết nối với bơm chân không (RZ 6, Vacuubrand, Đức). Quá trình sấy được thực hiện ở mức chân không cố định là -700 $\mathrm{mmHg}$ (tương ứng với áp suất tuyệt đối $60 \mathrm{mmHg}$ ) và bốn nhiệt độ sấy khác nhau $\left(40^{\circ} \mathrm{C}, 50^{\circ} \mathrm{C}, 60^{\circ} \mathrm{C}\right.$ và $\left.70^{\circ} \mathrm{C}\right)$. Độ ẩm ban đầu của mẫu được xác định là $78,68 \pm 1,09 \%$ (theo căn bản ướt) bằng phương pháp sấy ở $105^{\circ} \mathrm{C}$ đến khối lượng không đồi (Phạm Văn Sổ và Bùi Thị Như Thuận, 1991). Các trái cà chua được trải thành một lớp trên khay đựng mẫu và giữ bên trong buồng chân không. Hệ thống cần 3-5 phút để đạt độ chân không yêu cầu. Khối lượng mẫu được xác định sau mỗi 30 phút bằng cân phân tích (JJ200, G\&G, Trung Quốc, độ chính xác $\pm 0,01 \mathrm{~g}$ ) liên tục trong suốt quá trình sấy chân không đến khi đạt độ ẩm yêu cầu là $20 \%$ (tính theo căn bản ướt), tương ứng với giá trị hoạt độ nước $\mathrm{a}_{\mathrm{w}}$ của sản phẩm đạt khoảng 0,55 giúp hỗ trợ cho quá trình bảo quản vì giới hạn $\mathrm{a}_{\mathrm{w}}$ ức chế sự phát triển của nấm mốc và nấm men là khoảng 0,61 (Bhagwat, 2019). Thí nghiệm được thực hiện với ba lần lặp lại và số liệu biểu thị là giá trị trung bình.

\subsection{Mô hình hóa động học}

Tỷ lệ ẩm (MR - moisture ratio) của cà chua bi đen trong quá trình sấy chân không được tính toán theo công thức 1 (Akpinar, 2010).

$$
\mathrm{MR}=\frac{M_{t}-M_{e}}{M_{o}-M_{e}}
$$

Trong đó, $M R$ là tỷ lệ ẩm (không thứ nguyên); $M_{o}, M_{e}, M_{t}$ lần lượt là hàm lượng ẩm ban đầu, hàm lượng ẩm khi cân bằng và hàm lượng ẩm tại thời điểm $t(\mathrm{~kg}$ nước $/ \mathrm{kg}$ chất khô), $t$ là thời gian sấy (s).

Giả sử rằng hàm lượng ẩm ở bề mặt trái tương đương với hàm lượng ẩm cân bằng trong toàn bộ trái và bề mặt trái vẫn tiếp tục thoát ẩm trong suốt quá trình sấy $\left(\mathrm{M}_{\mathrm{e}} \approx 0\right)$ thì công thức 1 có thể được đơn giản hóa thành công thức 2 (Thakor et al., 1999).

$$
\mathrm{MR}=\frac{M_{t}}{M_{o}}
$$

\section{Bảng 1. Các mô hình đường cong sấy thông dụng}

\begin{tabular}{lll}
\hline Tên mô hình & Công thức & Tài liệu tham khảo \\
\hline Lewis & $\mathrm{MR}=\exp (-\mathrm{kt})$ & Bruce $(1985)$ \\
Page & $\mathrm{MR}=\exp \left(-\mathrm{kt}^{\mathrm{n}}\right)$ & Page $(1949)$ \\
Page điều chỉnh & $\mathrm{MR}=\exp \left[-(\mathrm{kt})^{\mathrm{n}}\right]$ & Overhults et al. $(1973)$ \\
Henderson và Pabis & $\mathrm{MR}=\mathrm{a} \exp (-\mathrm{kt})$ & Henderson and Pabis $(1961)$ \\
Logarit & $\mathrm{MR}=\mathrm{a} \exp (-\mathrm{kt})+\mathrm{c}$ & Toğrul and Pehlivan $(2002)$ \\
Hai tham số & $\mathrm{MR}=\mathrm{a} \exp \left(-\mathrm{k}_{\mathrm{o}} \mathrm{t}\right)+\mathrm{b} \exp \left(-\mathrm{k}_{\mathrm{t}} \mathrm{t}\right)$ & Henderson $(1974)$ \\
Hàm mũ hai tham số & $\mathrm{MR}=\mathrm{a} \exp (-\mathrm{kt})+(1-\mathrm{a}) \exp (-\mathrm{kat})$ & Sharaf-Eldeen et al. (1980) \\
Henderson và Pabis điều chỉnh & $\mathrm{MR}=\mathrm{a} \exp (-\mathrm{kt})+(1-\mathrm{a}) \exp (-\mathrm{kbt})$ & Yaldiz et al. $(2001)$ \\
\hline
\end{tabular}

Ghi chú: $k, k_{o}, k_{1}$ là các hằng số tốc độ sấy; $n, a$, b là các hệ số của mô hình 
Tám mô hình sấy thông dụng (Bảng 1) được phân tích thống kê bằng phần mềm Statgraphics Centurion XV (U.S.A.) để chọn ra mô hình tốt nhất mô tả đường cong sấy chân không của mẫu cà chua bi đen.

Hằng số tốc độ sấy và các hệ số của mô hình được xác định bằng phương pháp phân tích hồi quy phi tuyến tính (Thorat et al., 2012). Hệ số xác định $\left(\mathrm{R}^{2}\right)$ là chỉ tiêu quan trọng để lựa chọn mô hình tốt nhất mô tả đường cong sấy. Ngoài ra, giá trị $\chi^{2}$ là bình phương trung bình của độ lệch giữa giá trị thực nghiệm và giá trị tính toán và giá trị căn của sai số bình phương trung bình (root mean square error RMSE) cũng được sử dụng để xác định mức độ phù hợp của mô hình. Giá trị $\mathrm{R}^{2}$ càng cao và giá trị $\chi^{2}$ và RMSE càng thấp thì mức độ phù hợp càng tăng (Yaldýz and Ertekýn, 2001; Gunhan et al., 2005). Giá trị $\chi^{2}$ và RMSE được tính toán theo các công thức 3 và 4 (Akpinar, 2010; Zarein et al., 2015).

$$
\begin{aligned}
\chi^{2} & =\frac{\sum_{i=1}^{N}\left(\mathrm{MR}_{\exp , i}-\mathrm{MR}_{\mathrm{pre}, i}\right)^{2}}{N-Z} \\
\operatorname{RMSE} & =\left[\frac{1}{N} \sum_{i=1}^{N}\left(\mathrm{MR}_{\mathrm{pre}, i}-\mathrm{MR}_{\mathrm{exp}, i}\right)^{2}\right]^{1 / 2}
\end{aligned}
$$

Trong đó, $M R_{\text {exp }}$ là tỷ lệ ẩm không thứ nguyên thực nghiệm, $M R_{\text {pre }}$ là tỷ lệ ẩm không thứ nguyên dự đoán, $N$ là số điểm dữ liệu thực nghiệm và $z$ là số tham số trong mô hình.

\subsection{Tính toán độ khuếch tán ẩm hiệu quả và năng lượng hoạt hóa}

Độ khuếch tán ẩm hiệu quả của cà chua bi đen được ước tính bằng cách sử dụng mô hình khuếch tán của Fick (công thức 5) có tính đến sự co rút (Thorat et al., 2012).

$$
\frac{\partial u}{\partial t}=D_{e f f} \frac{\partial^{2} u}{\partial x^{2}}
$$

Trong đó, $u$ là nồng độ $\left(\mathrm{mol} / \mathrm{m}^{3}\right), D_{\text {eff }}$ là hệ số khuếch tán $\left(\mathrm{m}^{2} / \mathrm{s}\right), t$ là thời gian $(\mathrm{s}), x$ là độ dài khuếch tán $(\mathrm{m})$.

Trong trường hợp làm khô sản phẩm có dạng hình cầu, giả sử sự di chuyển ẩm theo một chiều mà độ khuếch tán không đổi trong toàn bộ trái, phân bố độ ẩm ban đầu đồng đều nhau, nồng độ bề mặt trái không đổi và sự co rút của cà chua bi hướng vào tâm trong quá trình sấy thì định luật này có thể được phát triển dưới dạng phương trình 6 được đề xuất bởi Crank (1979).

$$
\mathrm{MR}=\frac{6}{\pi^{2}} \sum_{n=1}^{\infty} \frac{1}{n^{2}} \exp \left(-n^{2} \pi^{2} \frac{D_{e f f} t}{r^{2}}\right)
$$

Trong đó $n$ là số nguyên dương, $r$ là bán kính trái có thể được biểu thị theo công thức 7 (An et al., 2013). Trong toàn bộ quá trình sấy, để tính đến sự co rút, bán kính trung bình của cà chua bi đen ở mồi khoảng thời gian $(0,5$ giờ $)$ được xác định.

$$
r=\sqrt[3]{\frac{3 V}{4 \pi}}
$$

Khi thời gian sấy dài $(\mathrm{n}=1)$, công thức 6 có thể được đơn giản hóa bằng cách lấy logarit của hai vế thành phương trình đường thẳng (8) (Doymaz, 2006).

$$
\ln (\mathrm{MR})=\ln \left(\frac{6}{\pi^{2}}\right)-\left(\frac{\pi^{2}}{r^{2}} D_{e f f} t\right)
$$

Độ khuếch tán ẩm được xác định bằng cách vẽ đồ thị của $\ln (\mathrm{MR})$ thực nghiệm theo $\left(\mathrm{t} / \mathrm{r}^{2}\right)$ bởi vì đồ thị cho một đường thẳng có hệ số góc theo công thức 9 (Doymaz, 2006).

$$
\text { Hệ số góc }=\pi^{2} D_{e f f}
$$

Năng lượng hoạt hóa được tính dựa vào phương trình Arrhenius (10) biểu diễn sự phụ thuộc của độ khuếch tán ẩm hiệu quả vào nhiệt độ (Sanjuán et al., 2003).

$$
D_{e f f}=D_{o} \exp \left(-\frac{E_{a}}{R T}\right)
$$

Trong đó, $D_{o}$ là thừa số Arrhenius (thừa số trước hàm mũ, $\mathrm{D}_{\mathrm{o}}$ tương đương với độ khuếch tán ở nhiệt độ cao vô hạn) $\left(\mathrm{m}^{2} / \mathrm{s}\right), E_{a}$ là năng lượng hoạt hóa $(\mathrm{kJ} / \mathrm{mol}), R$ là hằng số khí lý tưởng $(R=8,314$ $\mathrm{J} / \mathrm{mol} . \mathrm{K})$ và $T$ là nhiệt độ tuyệt đối (K).

\section{KẾT QUẢ VÀ THẢO LUẬN}

\section{1. Ảnh hưởng của nhiệt độ đến sự thay đổi độ ẩm theo thời gian sấy}

Quá trình sấy sẽ làm thay đổi độ ẩm của nguyên liệu. Độ ẩm giảm liên tục theo thời gian sấy, tuy nhiên, tốc độ mất ẩm diễn ra nhanh trong giai đoạn đầu của quá trình sấy do hàm lượng ẩm tự do trong nguyên liệu còn cao (Thorat et al., 2012) và sau đó tốc độ sấy giảm dần. Thời gian cần thiết để làm khô mẫu cà chua bi đen từ độ ầm ban đầu $78,68 \pm 1,09 \%$ (căn bản ướt) đến độ ẩm yêu cầu $20,00 \pm 1,17 \%$ (căn bản ướt) được rút ngắn khi tăng dần nhiệt độ sấy do quá trình truyền nhiệt và truyền khối diễn ra nhanh hơn (Thorat et al., 2012). Với nhiệt độ $40^{\circ} \mathrm{C}$, thời gian sấy nguyên liệu kéo dài đến 35 giờ để đạt độ ẩm yêu cầu, trong khi đó, thời gian sấy được rút ngắn còn 19 giờ, 11,5 giờ và 8 giờ khi tiến trình sấy được thực hiện ở nhiệt độ cao hơn (tương ứng với $\left.50,60,70^{\circ} \mathrm{C}\right) . \mathrm{Xu}$ hướng này cũng diễn ra tương tự 
khi sấy chân không các nguyên liệu khác như cà tím (Wu et al., 2007) và gừng (Thorat et al., 2012).

\subsection{Mô hình hóa đường cong sấy}

Các dữ liệu về tỷ lệ ẩm theo thời gian sấy sau đó được kiểm tra tính phù hợp với tám mô hình sấy thông dụng (Bảng 1). Kết quả phân tích thống kê được thể hiện ở Bảng 2. Giá trị $\mathrm{R}^{2}$ đều lớn hơn 0,97 cho thấy sự phù hợp tốt của tất cả các mô hình. Tính phù hợp của mô hình còn được đánh giá dựa trên giá trị $\chi^{2}$ và $R M S E$. Nhìn chung, giá trị $R^{2}, \chi^{2}$ và $R M S E$ dao động trong khoảng 0,9798 - 0,9995, 0,0000628 $-0,0020658$ và $0,0071913-0,0435160$. Kết quả tính toán cho thấy mô hình logarit thể hiện sự phù hợp cao hơn so với các mô hình khác ở tất cả các nhiệt độ sấy với $\mathrm{R}^{2}>0,998, \chi^{2}<0,0002$ và $\mathrm{RMSE}<0,02$. Hình 1 minh họa sự tương thích giữa mô hình logarit với kết quả thực nghiệm ở các nhiệt độ $40,50,60$ và $70^{\circ} \mathrm{C}$. Độ tin cậy của mô hình logarit còn được đánh giá bằng cách so sánh tỷ lệ ẩm tính toán ở các nhiệt độ sấy khác nhau với các giá trị tỷ lệ ẩm thu được từ dữ liệu thực nghiệm. Kết quả phân tích hồi quy tuyến tính cho thấy giá trị dữ liệu dao động gần đường thẳng có độ dốc $45^{\circ}$ chứng tỏ sự phù hợp của mô hình logarit trong việc mô tả quá trình sấy chân không cà chua bi đen, điển hình là số liệu thu được ở nhiệt độ $70^{\circ} \mathrm{C}$ với giá trị $\mathrm{R}^{2}$ đạt được là 0,9995 (Hình 2). Tương tự, ở các nhiệt độ còn lại $(40,50$ và $60^{\circ} \mathrm{C}$ ), giá trị $\mathrm{R}^{2}$ đạt được lần lượt là 0,$9986 ; 0,9988$ và 0,9989 .

\section{Bảng 2. Kết quả phân tích thống kê sự phù hợp của các mô hình với dữ liệu sấy}

\begin{tabular}{|c|c|c|c|c|c|c|c|}
\hline Mô hình & $\begin{array}{l}\text { Nhiệt độ } \\
\left.\text { sấy ( }{ }^{\circ} \mathrm{C}\right)\end{array}$ & \multicolumn{2}{|c|}{ Các hệ số của mô hình } & Hằng số tốc độ sấy & $\mathbf{R}^{2}$ & $\chi^{2}$ & RMSE \\
\hline \multirow{4}{*}{ Lewis } & 40 & & & $\mathrm{k}=0,0893$ & 0,9983 & 0,0001254 & 0,0111205 \\
\hline & 50 & & & $\mathrm{k}=0,1334$ & 0,9865 & 0,0011728 & 0,0338148 \\
\hline & 60 & & & $\mathrm{k}=0,1966$ & 0,9798 & 0,0018899 & 0,0425577 \\
\hline & 70 & & & $\mathrm{k}=0,2911$ & 0,9888 & 0,0010611 & 0,0316025 \\
\hline \multirow{4}{*}{ Page } & 40 & $\mathrm{n}=1,0256$ & & $\mathrm{k}=0,0836$ & 0,9983 & 0,0001136 & 0,0105059 \\
\hline & 50 & $\mathrm{n}=1,2040$ & & $\mathrm{k}=0,0860$ & 0,9975 & 0,0002032 & 0,0138828 \\
\hline & 60 & $\mathrm{n}=1,2606$ & & $\mathrm{k}=0,1258$ & 0,9957 & 0,0003829 & 0,0186968 \\
\hline & 70 & $\mathrm{n}=1,1570$ & & $\mathrm{k}=0,2359$ & 0,9938 & 0,0005220 & 0,0213719 \\
\hline \multirow{4}{*}{$\begin{array}{l}\text { Page điều } \\
\text { chỉnh }\end{array}$} & 40 & $\mathrm{n}=1,0255$ & & $\mathrm{k}=0,0889$ & 0,9985 & 0,0001119 & 0,0104305 \\
\hline & 50 & $\mathrm{n}=1,1339$ & & $\mathrm{k}=0,1403$ & 0,9949 & 0,0002037 & 0,0139012 \\
\hline & 60 & $\mathrm{n}=1,1493$ & & $\mathrm{k}=0,1897$ & 0,9961 & 0,0003827 & 0,0186925 \\
\hline & 70 & $\mathrm{n}=1,1983$ & & $\mathrm{k}=0,3087$ & 0,9928 & 0,0005226 & 0,0213830 \\
\hline \multirow{4}{*}{$\begin{array}{l}\text { Henderson } \\
\text { và Pabis }\end{array}$} & 40 & $a=1,0043$ & & $\mathrm{k}=0,0897$ & 0,9983 & 0,0001273 & 0,0111256 \\
\hline & 50 & $a=1,0567$ & & $\mathrm{k}=0,1410$ & 0,9906 & 0,0008640 & 0,0286494 \\
\hline & 60 & $a=1,0566$ & & $\mathrm{k}=0,2083$ & 0,9844 & 0,0015954 & 0,0382421 \\
\hline & 70 & $\mathrm{a}=1,0301$ & & $\mathrm{k}=0,3003$ & 0,9902 & 0,0010570 & 0,0305386 \\
\hline \multirow{4}{*}{ Logarit } & 40 & $a=1,0129$ & $c=-0,0169$ & $\mathrm{k}=0,0855$ & 0,9986 & 0,0001065 & 0,0101017 \\
\hline & 50 & $a=1,1577$ & $c=-0,1423$ & $\mathrm{k}=0,1038$ & 0,9988 & 0,0001196 & 0,0105165 \\
\hline & 60 & $a=1,2720$ & $c=-0,2653$ & $\mathrm{k}=0,1294$ & 0,9989 & 0,0001252 & 0,0104656 \\
\hline & 70 & $\mathrm{a}=1,1807$ & $c=-0,1883$ & $\mathrm{k}=0,2079$ & 0,9995 & 0,0000628 & 0,0071913 \\
\hline \multirow{4}{*}{$\begin{array}{l}\text { Hai tham } \\
\text { số }\end{array}$} & 40 & $a=0,5022$ & $\mathrm{~b}=0,5022$ & $\mathrm{k}_{\mathrm{o}}=0,0898 \quad \mathrm{k}_{1}=0,0896$ & 0,9983 & 0,0001349 & 0,0112880 \\
\hline & 50 & $a=0,5283$ & $\mathrm{~b}=0,5283$ & $\mathrm{k}_{\mathrm{o}}=0,1410 \quad \mathrm{k}_{1}=0,1410$ & 0,9906 & 0,0009627 & 0,0294345 \\
\hline & 60 & $\mathrm{a}=0,5283$ & $b=0,5283$ & $\mathrm{k}_{\mathrm{o}}=0,2082 \quad \mathrm{k}_{1}=0,2082$ & 0,9844 & 0,0019305 & 0,0401087 \\
\hline & 70 & $\mathrm{a}=0,5150$ & $\mathrm{~b}=0,5150$ & $\mathrm{k}_{\mathrm{o}}=0,3003 \quad \mathrm{k}_{1}=0,3003$ & 0,9902 & 0,0014072 & 0,0328038 \\
\hline \multirow{4}{*}{$\begin{array}{l}\text { Hàm mũ } \\
\text { hai tham } \\
\text { số }\end{array}$} & 40 & $a=0,0156$ & & $\mathrm{k}=5,6012$ & 0,9978 & 0,0001605 & 0,0112880 \\
\hline & 50 & $\mathrm{a}=1,0018$ & & $\mathrm{k}=0,1407$ & 0,9919 & 0,0001101 & 0,0102280 \\
\hline & 60 & $\mathrm{a}=0,9985$ & & $\mathrm{k}=0,1964$ & 0,9798 & 0,0020658 & 0,0435160 \\
\hline & 70 & $\mathrm{a}=0,9747$ & & $\mathrm{k}=0,2914$ & 0,9888 & 0,0012074 & 0,0326403 \\
\hline \multirow{4}{*}{$\begin{array}{l}\text { Henderson } \\
\text { và Pabis } \\
\text { điều chỉnh }\end{array}$} & 40 & $\mathrm{a}=1,15761$ & $b=0,5676$ & $\mathrm{k}=0,0813$ & 0,9966 & 0,0001070 & 0,0101309 \\
\hline & 50 & $\mathrm{a}=-2.3398$ & $b=1,3199$ & $\mathrm{k}=0,0547$ & 0,9968 & 0,0001223 & 0,0105924 \\
\hline & 60 & $a=2,6734$ & $\mathrm{~b}=0,5955$ & $\mathrm{k}=0,0950$ & 0,9970 & 0,0001274 & 0,0106935 \\
\hline & 70 & $a=0,3325$ & $\mathrm{~b}=1,0084$ & $\mathrm{k}=0,2854$ & 0,9935 & 0,0012876 & 0,0325629 \\
\hline
\end{tabular}




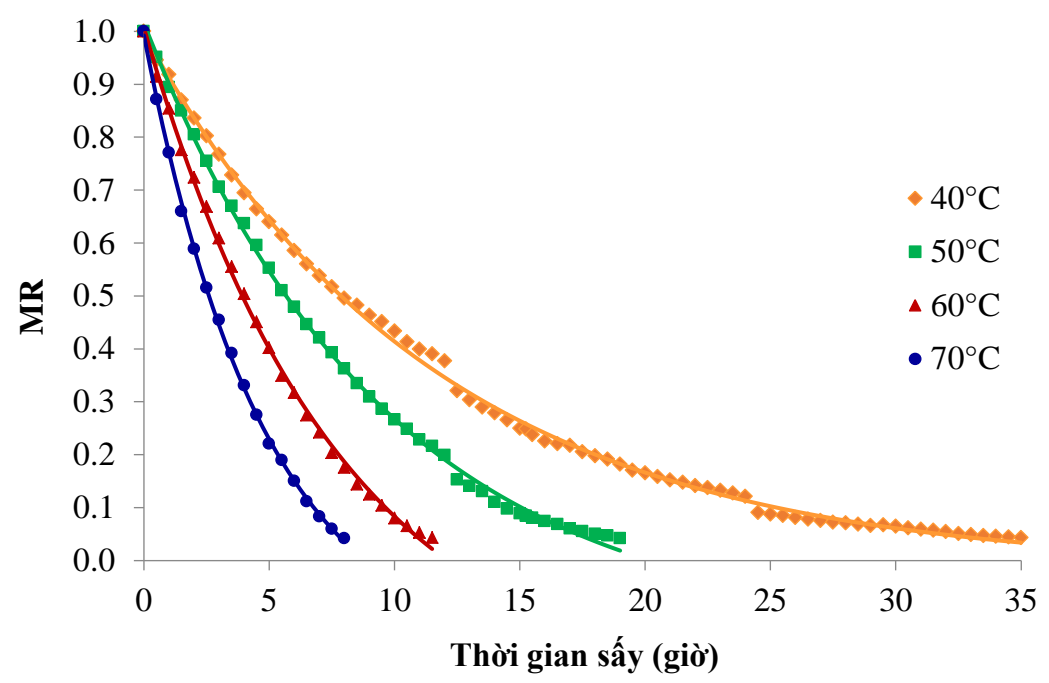

Hình 1. Tỷ lệ độ ẩm (MR) là hàm của thời gian sấy ở các nhiệt độ khác nhau

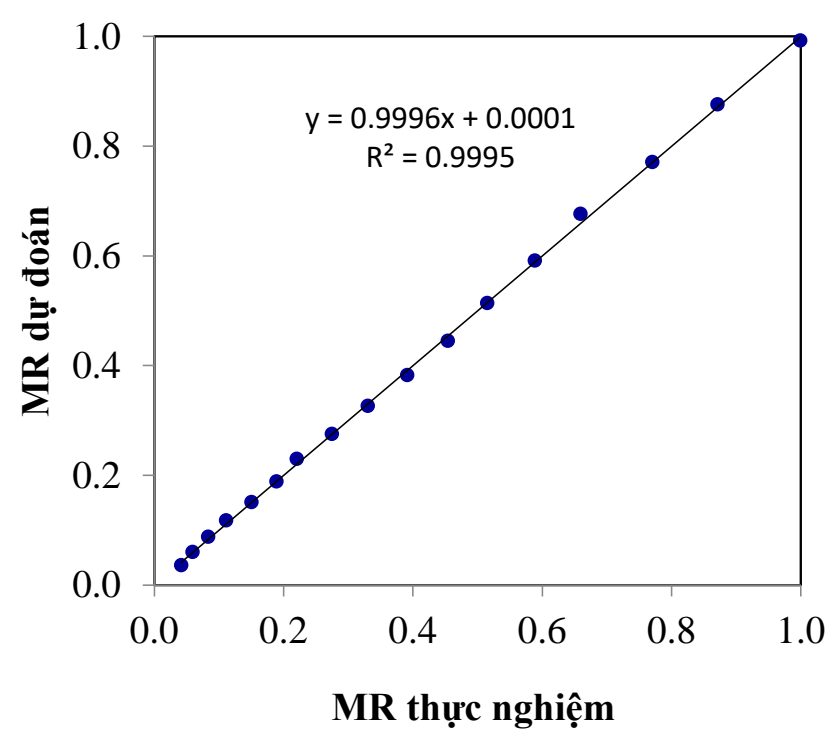

Hình 2. Tương thích giữa dữ liệu thực nghiệm và dữ liệu dự đoán theo mô hình logarit (trường hợp của tỷ lệ ẩm của cà chua bi đen sấy ở nhiệt độ $\mathbf{7 0}^{\circ} \mathrm{C}$ )

3.3. Độ khuếch tán ẩm hiệu quả và năng lượng hoạt hóa

Giá trị độ khuếch tán ẩm hiệu quả $\left(D_{\text {eff }}\right)$ của cà chua bi đen ở nhiệt độ $40-70^{\circ} \mathrm{C}$ dao động trong khoảng 3,9028.10-10 - 1,7580.10-9 $\mathrm{m}^{2} / \mathrm{s}$ (Bảng 3). Giá trị $D_{\text {eff }}$ tăng dần khi nhiệt độ tăng. Kết quả này thấp hơn so với các nghiên cứu trước đây khi sấy chân không cà tím $\left(D_{\text {eff }}=1,653-3,417 \cdot 10^{-9} \mathrm{~m}^{2} / \mathrm{s}\right)$ ở $30-50^{\circ} \mathrm{C}$ (Wu et al., 2007), cà rốt $\left(D_{\text {eff }}=1,474\right.$ $\left.2,740.10^{-9} \mathrm{~m}^{2} / \mathrm{s}\right)$ và bí đỏ $\left(D_{\text {eff }}=2,08-4,03^{-9} \mathrm{~m}^{2} / \mathrm{s}\right)$ ỏ $50-70^{\circ} \mathrm{C}$ (Arévalo-Pinedo and Murr, 2007), gừng $\left(D_{\text {eff }}=1,859-4,777 \times 10^{-8} \mathrm{~m}^{2} / \mathrm{s}\right)$ ơ $40-65^{\circ} \mathrm{C}$ (Thorat et al., 2012) do cà chua có lớp vỏ sáp hầu như không thấm nước, nước khuếch tán trong quá trình sấy chủ yếu xuyên qua các lỗ châm.

Bảng 3. Giá trị độ khuếch tán ẩm hiệu quả của trái cà chua bi đen ở các nhiệt độ sấy khác nhau

\begin{tabular}{cc}
\hline Nhiệt độ $\left({ }^{\mathbf{0}} \mathbf{C}\right)$ Độ khuếch tán ẩm hiệu quả $\left(\mathbf{m}^{\mathbf{2}} / \mathbf{s}\right)$ \\
\hline 40 & $3,9028.10^{-10}$ \\
50 & $7,5553.10^{-10}$ \\
60 & $1,2352.10^{-9}$ \\
70 & $1,7580.10^{-9}$ \\
\hline
\end{tabular}

Giá trị logarit của $D_{\text {eff }}$ được biểu diễn theo nghịch đảo của nhiệt độ tuyệt đối (Hình 3). Kết quả 
thể hiện mối quan hệ tuyến tính do sự phụ thuộc của độ khuếch tán ẩm vào nhiệt độ theo mô hình Arrhenius. Từ đó, năng lượng hoạt hóa tính được là $38,69 \mathrm{~kJ} / \mathrm{mol}$. Giá trị này cao hơn một ít so với năng lượng hoạt hóa đạt được khi sấy chân không gừng ở nhiệt độ $40-65^{\circ} \mathrm{C}(35,675 \mathrm{~kJ} / \mathrm{mol})$ (Thorat et al., 2012) nhưng thấp hơn so với giá trị đạt được khi sấy chân không vỏ quả lựu ở nhiệt độ $25-60^{\circ} \mathrm{C}(47,65$ $\mathrm{kJ} / \mathrm{mol}$ ) (Yilmaz et al., 2017).

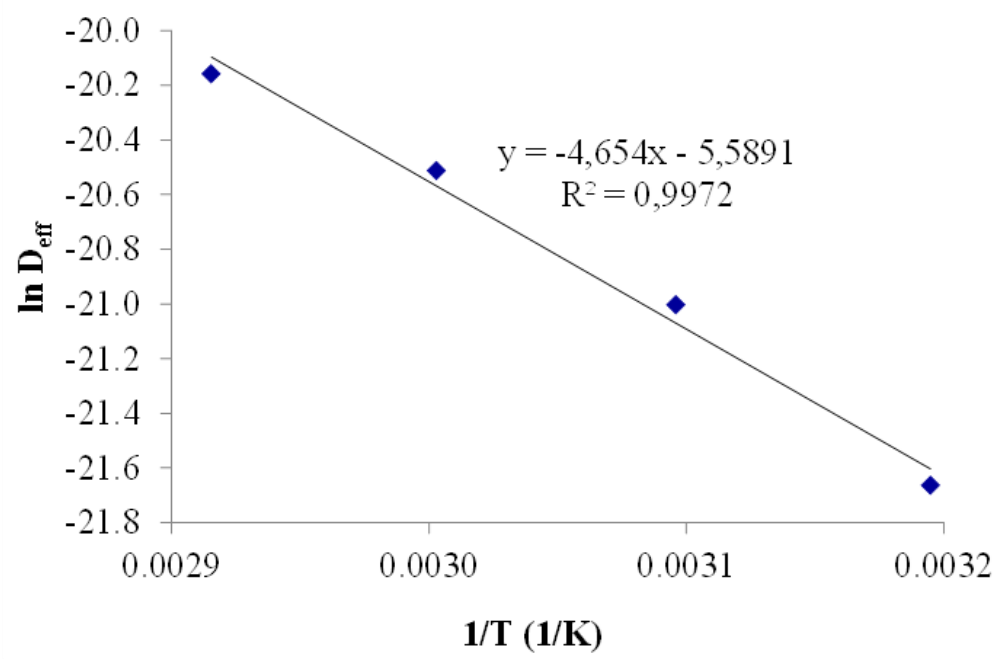

Hình 3. Mối quan hệ theo mô hình Arrhenius giữa độ khuếch tán ẩm hiệu quả và nhiệt độ

\section{KẾT LUẬN}

Trong số tám mô hình toán học được áp dụng, mô hình logarit được chọn để mô tả động học tiến trình sấy trái cà chua bi đen ở điều kiện chân không với độ tương thích cao hơn so với các mô hình còn lại ở các nhiệt độ sấy sử dụng $\left(\mathrm{R}^{2}>0,998, \chi^{2}<\right.$ $0,0002$ và $\mathrm{RMSE}<0,02)$. Độ khuếch tán ẩm hiệu quả thay đổi từ $3,9028 \cdot 10^{-10}$ đến $1,7580 \cdot 10^{-9}$. Sự phụ thuộc nhiệt độ của độ khuếch tán ẩm hiệu quả tuân theo phương trình Arrhenius với năng lượng hoạt hóa $38,69 \mathrm{~kJ} / \mathrm{mol}$. Sấy chân không ở nhiệt độ $70^{\circ} \mathrm{C}$ đã tỏ ra có hiệu quả trong việc sấy trái cà chua bi đen với thời gian kết thúc tiến trình sớm (8 giờ) để độ ẩm sản phẩm đạt yêu cầu ( $20 \%)$.

\section{TÀI LIỆU THAM KHẢO}

Akpinar, E. K. (2010). Drying of mint leaves in a solar dryer and under open sun: modelling, performance analyses. Energy Conversion and Management, 51(12), 2407-2418. https://doi.org/10.1016/j.enconman.2010.05.005

Alda, L. M., Gogoasa, I., Bordean, D.M., Gerden, I., Alda, S., Moldovan, C., \& Nita, L. (2009). Lycopene content of tomatoes and tomato products. Journal of Agroalimentary Processes and Technologies, 15(4), 540-542.

An, K., Li, H., Zhao, D., Ding, S., Tao, H., \& Wang, Z. (2013). Effect of osmotic dehydration with pulsed vacuum on hot-air drying kinetics and quality attributes of cherry tomatoes. Drying
Technology, 31(6), 698-706. https://doi.org/10.1080/07373937.2012.755192

Arévalo-Pinedo, A., \& Murr, F. E. X. (2007). Influence of pre-treatments on the drying kinetics during vacuum drying of carrot and pumpkin. Journal of Food Engineering, 80(1), 152-156. https://doi.org/10.1016/j.jfoodeng.2006.05.005

Bhagwat, V. R. (2019). Safety of water used in food production. In R. L. Singh, \& S. Mondal (Eds). Food Safety and Human Health (pp. 219247). Academic Press. https://doi.org/10.1016/C2017-0-04079-X

Bruce, D. M. (1985). Exposed-layer barley drying: three models fitted to new data up to 150 C. Journal of Agricultural Engineering Research, 32(4), 337-348. https://doi.org/10.1016/0021-8634(85)90098-8

Crank, J. (1979). The mathematics of diffusion. Oxford University Press. 414 pages.

Doymaz, I. (2006). Drying kinetics of black grapes treated with different solutions. Journal of Food Engineering, 76(2), 212-217. https://doi.org/10.1016/j.jfoodeng.2005.05.009

Ford, N. A., \& Erdman, J. W. (2012). Are lycopene metabolites metabolically active?. Acta Biochimica Polonica, 59(1). https://doi.org/10.18388/abp.2012_2159

Giri, S. K., \& Prasad, S. (2007). Drying kinetics and rehydration characteristics of microwavevacuum and convective hot-air dried 
mushrooms. Journal of Food Engineering, 78(2), 512-521. https://doi.org/10.1016/j.jfoodeng.2005.10.021

Gunhan, T., Demir, V., Hancioglu, E., \& Hepbasli, A. (2005). Mathematical modelling of drying of bay leaves. Energy Conversion and Management, 46(11-12), 1667-1679.

Ha, H. T. N., \& Thuy, N. M. (2020a). Effect of thermal treatment on quality of black cherry tomatoes (Solanum lycopersicum cv. OG): Optimization of the blanching parameters. International Journal of Agronomy and Agricultural Research, 16(4), 1-10.

Ha, H. T. N., \& Thuy, N. M. (2020b). Optimization of vacuum infiltration before blanching of black cherry tomatoes (Solanum lycopersicum cv. OG) using response surface methodology. Food Research, 4(4), 1317-1325. https://doi.org/10.26656/fr.2017.4(4).100

Henderson, S. M., \& Pabis, S. (1961). Grain drying theory I: Temperature effect on drying coefficient. Journal of AgriculturalEngineering Research. 7, 85-89.

Henderson, S. M. (1974). Progress in developing the thin layer drying equation. Transactions of American Society of Agricultural Engineers, 17(6), 1167-1172.

Ilahy, R., Hdider, C., \& Tlili, I. (2009). Bioactive compounds and antioxidant activity of tomato high lycopene content advanced breeding lines. The African Journal of Plant Science and Biotechnology, 3(1), 1-6.

Jaya, S., \& Das, H. (2003). A vacuum drying model for mango pulp. Drying Technology, 21(7, 12151234. https://doi.org/10.1081/DRT-120023177

Kaur, H., Chauhan, S., \& Sandhir, R. (2011). Protective effect of lycopene on oxidative stress and cognitive decline in rotenone induced model of Parkinson's disease. Neurochemical Research, 36(8), 1435-1443. https://doi.org/10.1007/s11064-011-0469-3

Li, H., Deng, Z., Liu, R., Young, J. C., Zhu, H., Loewen, S., \& Tsao, R. (2011). Characterization of phytochemicals and antioxidant activities of a purple tomato (Solanum lycopersicum L.). Journal of Agricultural and Food Chemistry, 59(21), 11803-11811. https://doi.org/10.1021/jf202364v

Lila, M.A. (2004). Anthocyanins and human health: an in vitro investigative approach. Journal of Biomedicine and Biotechnology, 2004(5), 306-313.

Michailidis, P. A., \& Krokida, M. K. (2014). Drying and dehydration processes in food preservation and processing. Conventional and Advanced Food Processing Technologies, 1-32. https://doi.org/10.1002/9781118406281.ch1
Min, J. Y., \& Min, K. B. (2014). Serum lycopene, lutein and zeaxanthin, and the risk of Alzheimer's disease mortality in older adults. Dementia and Geriatric Cognitive Disorders, 37(3-4), 246-256. https://doi.org/10.1159/000356486

Mordente, A. L. V. A. R. O., Guantario, B., Meucci, E., Mordente, A. L. V. A. R. O., Guantario, B., Meucci, E., Silvestrini, A., Lombardi, E., E Martorana, G., ... \& Bohm, V. (2011). Lycopene and cardiovascular diseases: an update. Current Medicinal Chemistry, 18(8), 1146-1163. https://doi.org/10.2174/092986711795029717

Overhults, D. G., White, G. M., Hamilton, H. E., \& Ross, I. J. (1973). Drying soybeans with heated air. Transactions of the ASAE, 16(1), 112. https://doi.org/10.13031/2013.37459

Page, G. E. (1949). Factors influencing the maximum rates of airdrying shelled corn in thin layers. M.S. thesis. Department ofMechanical Engineering, Purdue University, Purdue, USA.

Phạm Văn Sổ và Bùi Thị NhưThuận (1991). Kiểm nghiệm lương thực, thực phẩm. Trường $Đ H$ Bách khoa Hà Nội.

Sanjuán, N., Lozano, M., García-Pascual, P., \& Mulet, A. (2003). Dehydration kinetics of red pepper (Capsicum annuum $\mathrm{L}$ var Jaranda). Journal of the Science of Food and Agriculture, 83(7), 697-701. https://doi.org/10.1002/jsfa.1334

Sharaf-Eldeen, Y. I., Blaisdell, J. L., \& Hamdy, M.Y. (1980). A model for ear corn drying. Transactions of the ASAE, 5(4), 12611265.

Thakor, N. J., Sokhansanj, S., Sosulski, F. W., \& Yannacopoulos, S. (1999). Mass and dimensional changes of single canola kernels during drying. Journal of Food Engineering, 40(3), 153-160. https://doi.org/10.1016/S0260-8774(99)00042-4

Thorat, I. D., Mohapatra, D., Sutar, R.F., Kapdi, S. S., \& Jagtap, D. D. (2012). Mathematical modeling and experimental study on thin-layer vacuum drying of ginger (Zingiber officinale R.) slices. Food and Bioprocess Technology, 5(4), 1379-1383. https://doi.org/10.1007/s11947-010-0429-y

Toğrul, İ. T., \& Pehlivan, D. (2002). Mathematical modelling of solar drying of apricots in thin layers. Journal of Food Engineering, 55(3), 209216. https://doi.org/10.1016/S02608774(02)00065-1

Wang, Z. F., Fang, S. Z., \& Hu, X. S. (2009). Effective diffusivities and energy consumption of whole fruit Chinese jujube (Zizyphus jujuba Miller) in microwave drying. Drying Technology, 27(10), 1097-1104. https://doi.org/10.1080/07373930903221200 
Wu, L., Orikasa, T., Ogawa, Y., \& Tagawa, A. (2007). Vacuum drying characteristics of eggplants. Journal of Food Engineering, 83(3), 422-429.

https://doi.org/10.1016/j.jfoodeng.2007.03.030

Yaldiz, O., Ertekin, C., \& Uzun, H.I. (2001). Mathematical modeling of thin layer solar drying of sultana grapes. Energy, 26(5), 457-465. https://doi.org/10.1016/S0360-5442(01)00018-4

Yaldýz, O., \& Ertekýn, C. (2001). Thin layer solar drying of some vegetables. Drying Technology, 19(3-4), 583-597. https://doi.org/10.1081/DRT-100103936

Yılmaz, F. M., Yüksekkaya, S., Vardin, H., \& Karaaslan, M. (2017). The effects of drying conditions on moisture transfer and quality of pomegranate fruit leather (pestil). Journal of the Saudi Society of Agricultural Sciences, 16(1), 3340. https://doi.org/10.1016/j.jssas.2015.01.003

Zarein, M., Samadi, S. H., \& Ghobadian, B. (2015). Investigation of microwave dryer effect on energy efficiency during drying of apple slices. Journal of the Saudi Society of Agricultural Sciences, 14(1), 41-47. https://doi.org/10.1016/j.jssas.2013.06.002

Zhang, W., Xie, F., Lan, X., Gong, S., \& Wang, Z. (2018). Characteristics of pectin from black cherry tomato waste modified by dynamic highpressure microfluidization. Journal of Food Engineering, 216, 90-97. https://doi.org/10.1016/j.jfoodeng.2017.07.032 